\title{
Polytopic Control of a PV-fed SEPIC DC-DC Converter
}

\author{
Aleksandra Lekić* ${ }^{*}$ Abdelali El Aroudi ${ }^{\dagger}$, Dušan Stipanović ${ }^{\ddagger}$ \\ ${ }^{*}$ Department of Electronics, School of Electrical Engineering, University of Belgrade, \\ 11000 Belgrade, Serbia, Email: lekic.aleksandra@etf.bg.ac.rs \\ ${ }^{\dagger}$ Departament d'Enginyeria Electrònica, Elèctrica i Automàtica, Escola Tècnica Superior d'Enginyeria, \\ Universitat Rovira i Virgili, 43007 Tarragona, Spain \\ $\ddagger$ Coordinated Science Laboratory and ISE Department, University of Illinois at Urbana-Champaign, \\ Urbana, Illinois, USA
}

\begin{abstract}
In this paper, a polytopic control approach is applied to a PV-fed single-ended primary-inductance (SEPIC) dcdc converter. In a clear-cut-contrast with conventional control techniques based on averaging procedures, the polytopic control approach is applied to the switched model of the system. In particular, the switching control signal is synthesized using a Lyapunov function which has polytopic level sets. The validity of the approach is illustrated and verified using numerical simulations showing that the controller can achieve good performance in terms of fast recovery due to input voltage variations.

Index Terms-Polytopic control, SEPIC, PV systems.
\end{abstract}

\section{INTRODUCTION}

Dc-dc converters are widely used in many industrial applications. These switching systems perform power conversion from the input voltage to the output voltage according to the load requirements. The control circuit is designed to derive the power switches. Pulse Width Modulation (PWM) with natural sampling is the traditional technique for controlling dc-dc switching converters and it consists in imposing the duration during which a switch is maintained closed or open according to an appropriate compensated/filtered analog error signal by comparing it with a repetitive triangular or saw-tooth periodic signal [1]. High performance controllers for switching converters can be strongly affected by the model accuracy.

The control of these switched systems has attracted the interest of many researchers. Most of the approaches are based on averaging procedures hence obtaining a continuous-time model to which standard control techniques can be used after linearization and obtaining the small signal transfer functions in the Laplace domain [1]. However, the conventional control and analysis for switching converters involves considerable simplification of the problem leading to inaccurate conclusions about the real switched dynamics of the system although it can provide useful insights for the designer on the system performance in terms of system response. Therefore, using the averaged model for controller design, the performances are only guaranteed for the averaged dynamics and for a practical design they are a posteriori checked for the actual switched converter at a final design stage mainly by performing extensive time consuming trial and error numerical simulations to verify that the response of the switched system matches to some extent with the averaged one. Under large ripple conditions in the control signals, the averaged model inaccurately predicts its dc steady-state values as reported in [2]. Other approaches are based on discrete-time modeling. This is the case of almost all digital control techniques [3] and model predictive control [4] among others. Few are the control techniques that start from the real switched model of the system. This is the case of sliding mode control [5] and dwell-time or hybrid control [6], [7] among others.

Recently, with the development of advanced control techniques and the increased computational power provided by the available hardware, analysis and synthesis of power converters in a new perspective is possible. Owing to the existence of switching actions, power converters are hybrid systems in nature. More specifically, they feature different modes of operation depending on the state of the switches, which can be seen as discrete states, where each state has an associated continuous-time dynamics. Hence more precise modeling technique along with corresponding advanced control methods are needed to achieve higher performance. Polytopic control is another technique which is based on the switched model of the system for which suitable polytope is constructed [8]. Polytopic Lyapunov functions present multiple piecewise linear Lyapunov functions whose derivative is negative on the polytope facets. These concatenations of multiple linear functions are not differentiable everywhere, but they can be treated using Filippov's calculus for discontinuous differential equations. If the polytope is properly constructed, then the transient behavior of the switching system can be exactly determined and the ripple of the state variables can be limited to desired value. Namely, the hysteresis, which is constructed by polytope facets exactly corresponds to the reciprocal value of the maximum allowed ripple and as such it indirectly limits duty-ratio and the switching frequency of the converter in the steady-state.

Although there are multiple algorithms which can be used for the construction of the polytope [8], [9], most of the researches assumes that system switches between all stable subsystems [8]. However, dc-dc converters, depending on their circuit topology and operation mode, often switch between two or three subsystems one of them could be marginally 
unstable while the remaining ones are stable. Moreover, most of research studies on the control of switching converters focused on canonical low order converters such as Buck, Boost and Buck-Boost topologies. The single-ended primaryinductance converter (SEPIC) is a Buck-Boost derived dcdc topology that provides a positive regulated output voltage from either a smaller or a larger input voltage. This type of conversion is required in many applications such as in power factor correction circuits [10] and in PV systems [11]. Unfortunately, the SEPIC topology is difficult to control using linearized averaged models because it is a high order converter corresponding to different independent energy storage elements making its analysis and design using conventional approaches a challenging task.

Motivated by the aforementioned difficulties, the purpose of this paper is to employ the polytopic control approach for designing a controller for the PV-fed SEPIC converter. Working in Continuous Conduction Mode (CCM), the system switches between one marginally stable and one stable subsystems.

The rest of the paper is organized as follows. Section II provides the switched model of the system and all its operating regimes. In Section III, expressions for computing the polytopic Lyapunov coefficients are derived and the simulation results for the SEPIC converter controlled using polytopic Lyapunov functions are provided. Section IV provides some concluding remarks.

\section{THE SWITCHED MODEL OF THE SEPIC CONVERTER}

The SEPIC converter depicted in Fig. 1 can operate in four subsystems. Each of these subsystems can be described by linear ordinary differential equations in the following form:

$$
\dot{\mathbf{x}}=\mathbf{A}_{i} \mathbf{x}+\mathbf{b}_{i}
$$

where $\mathbf{x}=\left[i_{L 1}, i_{L 2}, v_{p v}, v_{C}\right]^{T}, \mathbf{A}_{i}$ is $4 \times 4$ matrix of the $i$-th subsystem and $\mathbf{b}_{i}$ is column vector corresponding to $i$-th subsystem, where $i \in\{1,2\}$. All the subsystems are linear and time invariant. The switching devices is transistor denoted in Fig. 1 as $\mathrm{S}$ and a diode $\mathrm{D}$. The state of these devices determine the operating subsystem.

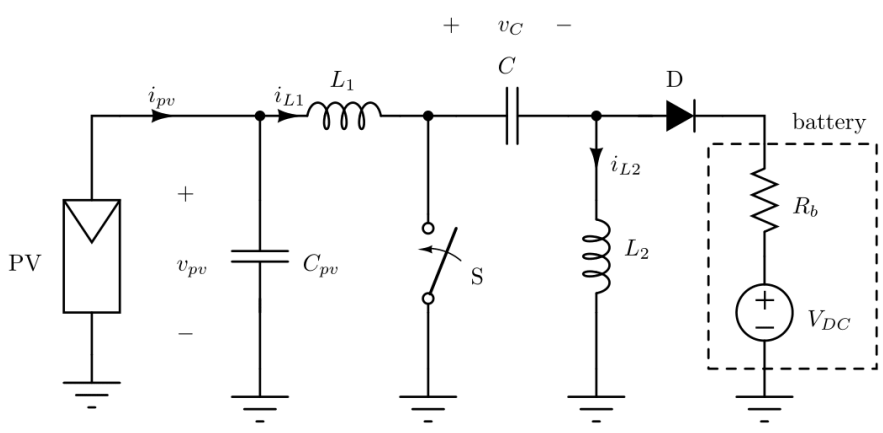

Fig. 1. PV system with the SEPIC converter.

The $i-v$ equation of the PV model can be approximated by the following linear Norton equivalent model:

$$
i_{\mathrm{pv}} \approx I_{\mathrm{mpp}}+G_{p N}\left(v_{\mathrm{pv}}-V_{\mathrm{mpp}}\right),
$$

where $G_{p N}=\partial i_{\mathrm{pv}} / \partial v_{\mathrm{pv}}$ is the equivalent Norton conductance and $I_{\mathrm{mpp}}$ and $V_{\mathrm{mpp}}$ are the generator current and voltage at the MPP (See [15] for more details). From (2), the equivalent Norton current $i_{p N}$ is as follows:

$$
i_{p N}=I_{\mathrm{mpp}}-G_{p N} V_{\mathrm{mpp}}
$$

By substituting the model of the PV source by its Norton equivalent, the following subsystems are obtained:

Subsystem 1, determined with switch $S$ turned ON and diode D turned OFF, is described in state-space using matrices

$$
\mathbf{A}_{1}=\left[\begin{array}{cccc}
0 & 0 & \frac{1}{L_{1}} & 0 \\
0 & 0 & 0 & -\frac{1}{L_{2}} \\
-\frac{1}{C_{p v}} & 0 & -\frac{G_{p N}}{C_{p v}} & 0 \\
0 & \frac{1}{C} & 0 & 0
\end{array}\right], \mathbf{b}_{1}=\left[\begin{array}{c}
0 \\
0 \\
\frac{i_{p N}}{C_{p v}} \\
0
\end{array}\right],
$$

for the state variables $\mathbf{x}=\left[\begin{array}{llll}i_{L 1} & i_{L 2} & v_{C 1} & v_{C 2}\end{array}\right]^{T}$. Subsystem 1 is marginally stable with all four eigenvalues having zero real part: $\lambda_{1,2}= \pm \frac{j}{\sqrt{L_{1} C_{p v}}}$ and $\lambda_{3,4}= \pm \frac{j}{\sqrt{L_{2} C}}$.

In subsystem 2 the switch $\mathrm{S}$ is OFF and diode D is ON, so the system matrices become

$$
\mathbf{A}_{2}=\left[\begin{array}{cccc}
-\frac{R_{b}}{L_{1}} & \frac{R_{b}}{L_{1}} & \frac{1}{L_{1}} & -\frac{1}{L_{1}} \\
\frac{R_{b}}{L_{2}} & -\frac{R_{b}}{L_{2}} & 0 & 0 \\
-\frac{1}{C_{p v}} & 0 & -\frac{G_{p N}}{C_{p v}} & 0 \\
\frac{1}{C_{1}} & 0 & 0 & 0
\end{array}\right], \mathbf{b}_{2}=\left[\begin{array}{c}
-\frac{V_{D C}}{L_{1}} \\
\frac{V_{D C}}{L_{2}} \\
\frac{i_{p N}}{C_{p v}} \\
0
\end{array}\right] .
$$

This subsystem is also marginally stable with one zero eigenvalue and three eigenvalues with negative real parts. Assuming CCM operation, only the previous two subsystems will be used in the control design.

The system is controlled by applying the PWM signal $q$ on the switch $\mathrm{S}$. The synthesis of this signal will be performed using the polytopic control and hysteretic frequency modulation. Other fixed frequency modulation strategies such as peak current control can also be used.

If we assume that the maximum power point of the PV panel is denoted as $I_{\mathrm{mpp}}$ and $V_{\mathrm{mpp}}$, then the desired equilibrium point, or the steady-state values of the averaged state variables during one switching period $T[1]$, is

$$
\overline{\mathbf{x}}=\left[\begin{array}{c}
\overline{i_{L 1}} \\
\overline{i_{L 2}} \\
\overline{v_{p v}} \\
\overline{v_{C}}
\end{array}\right]=\left[\begin{array}{c}
I_{\mathrm{mpp}} \\
-\frac{1-D}{D} I_{\mathrm{mpp}} \\
V_{\mathrm{mpp}} \\
V_{\mathrm{mpp}}
\end{array}\right],
$$

for a duty-ratio

$$
D=\frac{\frac{V_{D C}}{R_{b}}-I_{\mathrm{mpp}}+\sqrt{\left(\frac{V_{D C}}{R_{b}}-I_{\mathrm{mpp}}\right)^{2}+4 I_{\mathrm{mpp}} \frac{V_{\mathrm{mpp}}+V_{D C}}{R_{b}}}}{2 \frac{V_{\mathrm{mpp}}+V_{D C}}{R_{b}}} .
$$

Since in power electronics circuits the time constants of the reactive elements are much greater than the switching period, linear ripple approximation can be used and hence the state variables can be presented as linearly increasing or decreasing during the switching period $T$. For the PV-fed 
SEPIC converter, the steady-state ripple of the different state variables is given by the following expressions:

$$
\begin{aligned}
& \Delta i_{L 1}=\frac{V_{\mathrm{mpp}} D T}{L_{1}}, \quad \Delta i_{L 2}=\frac{V_{\mathrm{mpp}} D T}{L_{2}}, \\
& \Delta v_{p v}=\frac{V_{\mathrm{mpp}} D T^{2}}{8 L_{1} C_{p v}}, \Delta v_{C}=\frac{(1-D) I_{\mathrm{mpp}} T}{C} .
\end{aligned}
$$

The ripple vector of the state variables is then given by $\Delta \mathbf{y}=\left[\Delta i_{L 1}, \Delta i_{L 2}, \Delta v_{p v}, \Delta v_{C}\right]^{T}$.

\section{POLYTOPIC LYAPUNOV FUNCTION}

In this section it will be shown that the PV-fed SEPIC converter can be controlled using polytopic Lyapunov functions. If we construct a valid polytope and apply piecewise linear Lyapunov functions as in [14], then the obtained control stabilizes the system. First, let us recall the form of the polytopic Lyapunov function with the respect to $\mathbf{y}=\mathbf{x}-\overline{\mathbf{x}}$ :

$$
V(\mathbf{y})=\max _{i \in \mathbf{J}}\left|k_{i} y_{i}\right|, \quad \mathbf{J}=\{1,2,3,4\},
$$

and suppose that $V(\mathbf{y})$ as defined in (9) has a maximum value for the state variable labeled as $j$. Then the corresponding derivative for the interior points is

$$
\dot{V}_{i}(\mathbf{y})=k_{j} \operatorname{sgn}\left(k_{j} y_{j}\right)\left(\mathbf{A}_{i}\langle j, 1: 4\rangle \mathbf{y}+\overline{\mathbf{B}}_{i}\langle j\rangle\right)
$$

for the $i$-th subsystem in which $\overline{\mathbf{B}}_{i}=\mathbf{b}_{i}+\mathbf{A}_{i} \overline{\mathbf{x}}$. The attractive set can be expressed in the following form:

$$
\mathbf{P}=\left\{\mathbf{y} \in \mathbb{R}^{4}:\left|k_{j} y_{j}\right| \leq 1, j \in \mathbf{J}\right\} .
$$

Then, a stabilizing switching rule, we propose, can be formulated as follows:

$$
q= \begin{cases}0 & \text { if } 1 \text { and } k_{j} y_{j} \geq 1 \\ \{0,1\} & \text { if }\{0,1\} \text { and }-1<k_{j} y_{j}<1 \\ 1 & \text { if } 0 \text { and } k_{j} y_{j} \leq-1\end{cases}
$$

and

$$
j=\arg \max _{i \in \mathbf{J}}\left|k_{i} y_{i}\right| .
$$

The switching rule from equation (12) results in hysteresis control depicted in Fig. 2, where $k_{j} y_{j}$ correspond to the polytope facet for which is satisfied equation (13).

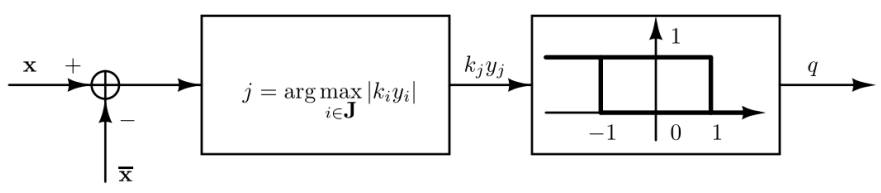

Fig. 2. Polytopic switching law.

In order to obtain stable bounded system polytope from the equation (11), using the ripple equation (8), we can determine that coefficients must satisfy the following limits:

$$
\begin{aligned}
& k_{1}=\frac{L_{1}}{\rho}>0, \quad\left|k_{2}\right|<\frac{L_{2}}{\rho}, k_{2}<0, \\
& \left|k_{3}\right|<\frac{8 L_{1} C_{p v}}{T \rho}, k_{3}<0, \quad k_{4}=-\frac{D V_{\mathrm{mpp}} C}{(1-D) I_{\mathrm{mpp}} \rho}<0,
\end{aligned}
$$

where $\rho=D V_{\mathrm{mpp}} T / 2$. The coefficients $k_{1}$ and $k_{3}$ of the polytope $\mathbf{P}$ correspond to the unstable variable $y_{1}$ and the variable $y_{3}$ directly dependent on $y_{1}$ in subsystem 1 . Thus, the state variables $y_{1}$ and $y_{3}$ are crucial for determining the hysteresis size during system's operation. For the derivation of equation (14) we used approach presented in Theorem 1 in [14]. In [14], it is showed that for the careful chosen polytope coefficients, the converter controlled using polytopic Lyapunov functions is stable and robust.

\section{NUMERICAL SIMULATION RESULTS}

Basically the main outcome of this paper is that the polytopic Lyapunov functions, with the polytopic value sets defined in equations (11) and (14), can be used for the control of PVfed SEPIC converter. For the illustration purposes we consider a SEPIC converter with parameters $L_{1}=L_{2}=580 \mu \mathrm{H}$, $C=1 \mu \mathrm{F}, C_{p}=20 \mu \mathrm{F}$, battery has parameters: $V_{D C}=42 \mathrm{~V}$ and $R_{b}=0.1 \Omega$, and the solar panel BP585, for which we construct the corresponding polytopic Lyapunov function. In Fig. 3, we depict the simulation results for the switching period $T=10 \mu \mathrm{s}$ and the PV panel operating at the temperature of $25^{\circ}$ with the irradiance $S=500 \mathrm{~W} / \mathrm{m}^{2}$ (with the maximum power $P_{\max }=42.56 \mathrm{~W}$ ) for the first $250 \mathrm{~ms}$ after which irradiance changes to the value $S=1000 \mathrm{~W} / \mathrm{m}^{2}$ (with $P_{\max }=84.96 \mathrm{~W}$ ).

During the first irradiance level $S=500 \mathrm{~W} / \mathrm{m}^{2}$ the PV panel has the maximum power point (MPP) for $V_{\mathrm{mpp}}=18 \mathrm{~V}$ and $I_{\mathrm{mpp}}=2.36 \mathrm{~A}$. Using equations (6) and (7) we estimate duty-ratio and equilibrium in the MPP steady-state as: $D=0.7017$ and $\overline{\mathbf{x}}=[2.3600 \mathrm{~A},-1.0034 \mathrm{~A}, 18 \mathrm{~V}, 18 \mathrm{~V}]^{T}$. Polytopic control is constructed to satisfy equation (14) with $\rho=6.3151 \times 10^{-5}$ and coefficients given by equation:

$$
\mathbf{k}=\left[\begin{array}{l}
k_{1} \\
k_{2} \\
k_{3} \\
k_{4}
\end{array}\right]=\left[\begin{array}{c}
\frac{L_{1}}{\rho} \\
-\frac{L_{2}}{10 \rho} \\
-\frac{L_{1} C_{p v}}{5 T \rho} \\
-\frac{D V_{\mathrm{mpp}} C}{(1-D) I_{\mathrm{mpp}} \rho}
\end{array}\right],
$$

which for the specified parameters has value: $\mathbf{k}=$ $\left[9.1844 \mathrm{~A}^{-1} ;-0.9184 \mathrm{~A}^{-1} ;-3.6738 \mathrm{~V}^{-1} ;-0.2841 \mathrm{~V}^{-1}\right]^{T}$.

After changing irradiance to the value $S=1000 \mathrm{~W} / \mathrm{m}^{2}$, $\mathrm{PV}$ panels MPP becomes $V_{\mathrm{p}}=18 \mathrm{~V}$ and $I_{\mathrm{p}}=4.72 \mathrm{~A}$ with a steady-state: $D=0.7299$ and $\overline{\mathbf{x}}=[4.72 \mathrm{~A}, \quad-1.991 \mathrm{~A}, \quad 18 \mathrm{~V}, \quad 18 \mathrm{~V}]^{T}$. Polytopic control is then defined by a hysteresis parameter $\rho=6.3299 \times 10^{-5}$ and the coefficients $\mathbf{k}=$ $\left[9.1629 \mathrm{~A}^{-1} ;-0.9163 \mathrm{~A}^{-1} ;-3.6652 \mathrm{~V}^{-1} ;-0.1428 \mathrm{~V}^{-1}\right]^{T}$.

In Fig. 3(a), the time domain response of the PV-fed SEPIC converter at startup and under irradiance change is depicted. This variation in this weather conditions parameter changes many other related parameters such as the maximum power point voltage, the maximum power point current, the Norton equivalent conductance and the operating duty cycle.

It can be observed that after a transient period, the system operation converges to the maximum power point. Under irradiance change, the system responds well to the desired 


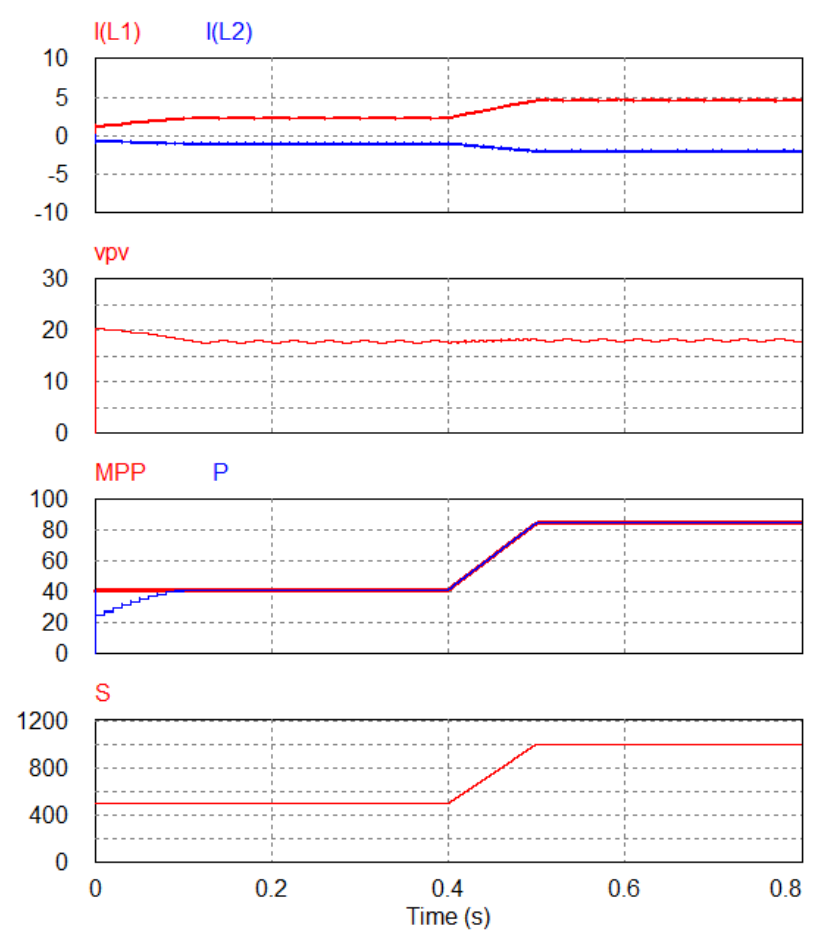

(a) With full-order feedback

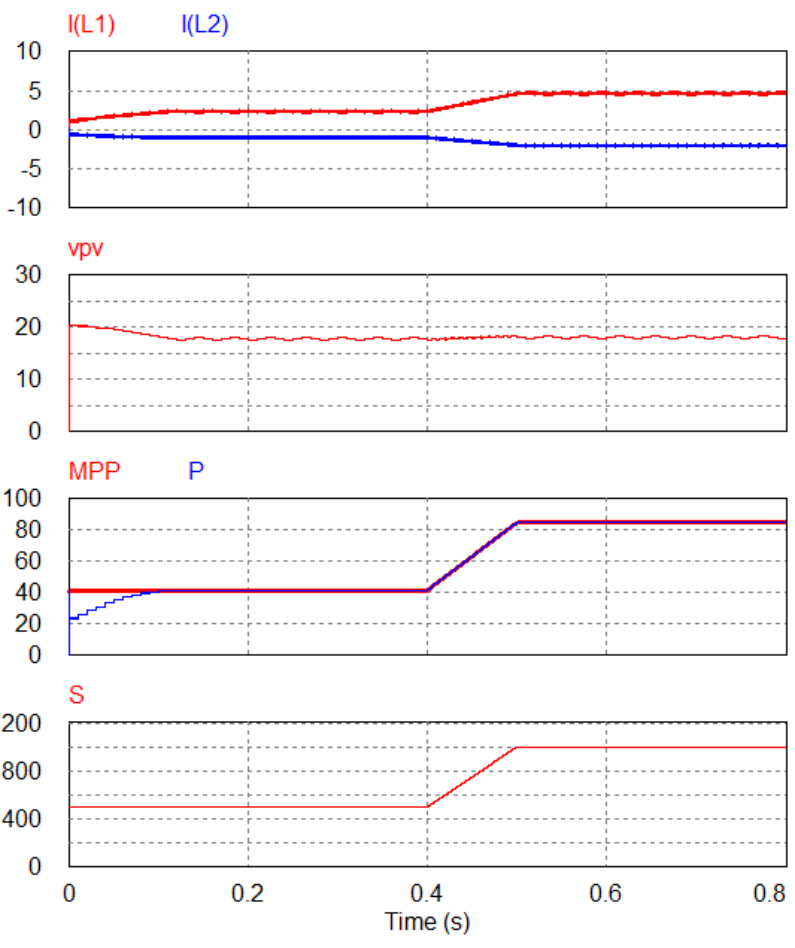

(b) With reduced-order feedback

Fig. 3. SEPIC converters time-domain response with the derived control using polytopic Lyapunov functions applied to the PV-fed SEPIC converter with a Perturb and Observ MPPT controller. During the first $250 \mathrm{~ms}, S=500 \mathrm{~W} / \mathrm{m}^{2}$. At $t=250 \mathrm{~ms}$, the irradiance changes to $S=1000 \mathrm{~W} / \mathrm{m}^{2}$.

voltage and current levels are imposed by the Perturb and Observe MPPT controller whose parameters were sampling period $T_{\mathrm{mppt}}=10 \mathrm{~ms}$ and perturbation $\Delta I_{\mathrm{mpp}}=0.2 \mathrm{~A}$.

It is worth noticing that the polytopic control is a very attractive approach because it results on precise computation of the required feedback gains for ensuring stability and robustness of the real switched system. However, it leads to a full-order state feedback whose implementation is possible only when all the needed state variables are measurable. Even under this condition, the number of required sensors for all the state variables is relatively high. In practice, either only a limited number of state variables may be measurable or there are a strict constraint on the number of the requested sensors due to cost considerations. In such cases, some solutions can be used to avoid the use of the full-order state feedback imposed by the polytopic control. One solution could be the use of state observers. Another more simple solution is to relax the controller by removing those components that have weak contribution to the synthesized control law. From the numerical values of the coefficients in the vector $\mathbf{k}$, it can be observed that the most important coefficients are the one corresponding to the inductor current $i_{L 1}$ and the capacitor voltage $v_{p v}$. In Fig. 3(b), the system is simulated under the same conditions corresponding to Fig. 3(a) but now by using only the inductor current $i_{L 1}$ and the capacitor $v_{p v}$ in the feedback. It can be observed that the results in terms of maximum power point tracking, stability and robustness are barely changed.

\section{CONClusion}

In this paper, a control regulation design for the SEPIC converter is proposed and and is based on carefully constructed polytopic Lyapunov functions. Due to the complexity of the SEPIC converter, the constructed polytopic Lyapunov functions have specific coefficients' values. This means that the coefficients determining polytope facets, which correspond to the unstable subsystem's variables are the dominant ones and these facets determine the ripple size of the state variables. The proposed regulator design is robust and has fast recovery time with respect to the change of the load and input voltage.

\section{ACKNOWLEDGMENTS}

This work was partially supported by the Ministry of Education, Science and Technological Development of the Republic of Serbia through project TR33020, by the Spanish Agencia Estatal de Investigación (AEI) and the Fondo Europeo de Desarrollo Regional (FEDER) under grant DPI2017-84572C2-1-R (AEI/FEDER, UE) and also by the Spanish Ministerio de Economía y Competitividad, in the frame of project MTM2015-65608-P, and by the National Science Foundation under Grant No. 1528036 (NRI: ASPIRE: Automation Supporting Prolonged Independent Residence for the Elderly).

\section{REFERENCES}

[1] R. W. Erickson and D. Maksimovic, Fundamentals of power electronics. Springer Science \& Business Media, 2001. 
[2] Lehman B. and Bass R. M., "Switching Frequency Dependent Averaged Models for PWM dc-dc Converters," IEEE Transactions on Power Electronics, vol. 11, no. 1, pp. 89-98, 1996.

[3] A. Prodić and D. Maksimović, "Digital PWM controller and current estimator for a low-power switching converter," The 7th Workshop on Computers in Power Electronics, COMPEL 2000, pp. 123-128, 2000.

[4] A.G. Beccuti, S. Mariéthoz, S. Cliquennois, S. Wang and M. Morari, "Explicit model predictive control of dc-dc switched-mode power supplies with extended Kalman filtering," IEEE Transactions on Industrial Electronics, vol. 56, no. 6, pp. 1864-1874, 2009.

[5] J. Calvente, A. El Aroudi, R. Giral, A. Cid-Pastor, E. Vidal-Idiarte, "Design of Current Programmed Switching Converters Using SlidingMode Control Theory," energies, vol. 11 no. 8, 2034, 2018.

[6] Chesi, P. Colaneri, J. C. Geromel, R. Middleton and R. Shorten, "Computing upper-bounds of the minimum dwell time of linear switched systems via homogeneous polynomial Lyapunov functions," Proceedings of the 2010 American Control Conference, Baltimore, MD, pp. 2487$2492,2010$.

[7] G. S. Deaecto, J. C. Geromel, F. S. Garcia and J. A. Pomilio, "Switched affine systems control design with application to dc-dc converters," IET Control Theory \& Applications, vol. 4, no. 7, pp. 1201-1210, July 2010.

[8] Y. Ohta, H. Imanishi, L. Gong and H. Haneda, "Computer generated Lyapunov functions for a class of nonlinear systems," IEEE Transactions on Circuits and Systems I: Fundamental Theory and Applications, vol. 40, no. 5, pp. 343-354, May 1993.

[9] F. Blanchini, "Nonquadratic Lyapunov functions for robust control," Automatica, vol. 31, no. 3, pp. 451-461, 1995.

[10] M. Bodetto, A. E. Aroudi, A. Cid-Pastor and M. S. Al-Numay, "Improving the Dimming Performance of Low-Power Single-Stage AC-DC HBLED Drivers," IEEE Transactions on Industrial Electronics, vol. 64, no. 7, pp. 5797-5806, July 2017.

[11] S. J. Chiang, H. Shieh and M. Chen, "Modeling and Control of PV Charger System With SEPIC Converter," IEEE Transactions on Industrial Electronics, vol. 56, no. 11, pp. 4344-4353, Nov. 2009.

[12] H. K. Khalil, "Noninear systems," Prentice-Hall, New Jersey, vol. 2, no. 5 , pp. 5-1, 1996

[13] A. Lekić and D. M. Stipanović, "Hysteresis switching control of the Cuk converter," IEEE Transactions on Circuits and Systems I: Regular Papers, vol. 63, no. 11, pp. 2048-2061, Nov. 2016.

[14] A. Lekić, D. Stipanović, and N. Petrović, "Controlling the Ćuk converter using polytopic Lyapunov functions," IEEE Transactions on Circuits and Systems II: Express Briefs, vol. 65, no. 11, pp. 1678 - 1682, Nov. 2018.

[15] A. El Aroudi, M. Al-Numay, G. Garcia, K. Al Hossani, N. Al Sayari and A. Cid-Pastor "Analysis of Nonlinear Dynamics of a Quadratic Boost Converter Used for Maximum Power Point Tracking in a Grid-Interlinked PV System,", Energies, vol. 12, no. 1, 61, 2019; doi:10.3390/en12010061. 